\title{
Duration of Cessation of Smoking before Elective Surgery: Impact on Intraoperative Hemodynamics and Early Postoperative Pain in Developing Country
}

\author{
Mohsin Nazir', Gauhar Afshan ${ }^{*}$, Muslima Ejaz², Amir Raza1 \\ ${ }^{1}$ Department of Anaesthesiology, Aga Khan University, Karachi, Pakistan \\ ${ }^{2}$ Department of Community Health Sciences, Aga Khan University, Karachi, Pakistan \\ Email: *gauhar.afshan@aku.edu
}

How to cite this paper: Nazir, M., Afshan, G., Ejaz, M. and Raza, A. (2021) Duration of Cessation of Smoking before Elective Surgery: Impact on Intraoperative Hemodynamics and Early Postoperative Pain in Developing Country. Open Journal of Anesthesiology, 11, 288-297.

https://doi.org/10.4236/ojanes.2021.119028

Received: May 22, 2021

Accepted: September 25, 2021

Published: September 28, 2021

Copyright $\odot 2021$ by author(s) and Scientific Research Publishing Inc. This work is licensed under the Creative Commons Attribution International License (CC BY 4.0).

http://creativecommons.org/licenses/by/4.0/

(c) (i) Open Access

\begin{abstract}
Aim and Background: It is estimated that up to $20 \%$ of patients coming for elective surgery are smokers and carry a risk of perioperative complications. Though smoking cessation and its impact on perioperative outcome are widely investigated worldwide we were unable to find any data in Pakistan. The objective of the study is to determine the impact of the duration of smoking cessation before elective surgery on intraoperative hemodynamics and postoperative pain in Pakistani population. Methods: It was a prospective cohort study conducted at the Aga Khan University Hospital Karachi, Pakistan, for one-year duration. A total of 260 patients scheduled for elective noncardiac surgery under general anaesthesia were recruited. Surgery under regional anaesthesia and minor surgery under general anaesthesia were excluded. Data on self-reported duration of smoking cessation by patients, intraoperative haemodynamics, postoperative pain scores and duration of hospital stay were collected by independently trained data collectors from the preoperative area until the patient is discharged from the hospital. Results: A data from 256 patients were analyzed. On the basis of self-reported duration of preoperative smoking cessation, patients were divided into 4 groups (Group 1: less than 2 days, Group 2: more than 2 days to 7 days, Group 3: more than 7 days to 4 weeks and Group 4: more than 4 weeks). It was found that the longer the duration of cessation of smoking is the less haemodynamic changes and lower postoperative pain scores. Length of stay did not show any difference among all four groups. No major postoperative pulmonary complication was found in any study patient. Conclusions: Duration of cessation of smoking before elective surgery is a significant predictor of intraoperative
\end{abstract}


haemodynamics and early postoperative pain in Pakistani population. A short duration of smoking cessation also helps to avoid some of the adverse effects and reduces perioperative complications.

\section{Keywords}

Preoperative Smoking Abstinence, Haemodynamic Variability,

Postoperative Pain, Smokers

\section{Introduction}

The global prevalence of smoking varies from less than $20 \%$ to more than $50 \%$. In Pakistan the prevalence of smoking among young males and females varies from $36 \%$ to $9 \%$ [1]. A study conducted in Karachi, the largest city of Pakistan and seventh largest city in the world, showed the prevalence of smoking is $32.7 \%$ [2]. It is estimated that up to $20 \%$ of patients coming for elective surgery are smokers and they carry a burden of comorbidities and risk of overall perioperative complications [3] [4] [5].

In current practice, smoking cessation of four weeks before an elective surgery has been recommended to reduce perioperative complications [3]. American and Canadian Society of Anesthesiologists has recommended smoking cessation of 4 - 8 weeks before any elective surgery [3]. However, a meta-analysis concluded that smoking cessation less than eight weeks before elective surgery did not affect the overall outcome [6].

It has been shown that the longer the duration of smoking cessation before surgery, the greater the benefit. Cessation of smoking for $2-4$ weeks prior to a planned elective surgery decreases the airway secretions \& reactivity and suppresses the hemodynamic response to laryngoscopy \& tracheal intubation while eight weeks of smoking cessation decreases the overall post-op morbidity and mortality [7]. It is likely that even a short duration of smoking cessation may give some benefit, considering the acute effects of nicotine and carbon monoxide on the cardiovascular system. It is also evident that cessation of smoking shortly before surgery, i.e. 48 - 72 hours decreases the carboxyhemoglobin levels (normal $<1.5 \%$, smokers $3 \%-10 \%)$ and increases the tissue oxygenation [8]. Considering the current evidence, it is important to note that the effective duration of smoking cessation is not yet determined and the precise effects of smoking cessation shortly before surgery are still unclear [7].

In Pakistan, it is a very common observation that the majority of smokers do not stop smoking 3 - 4 weeks before surgery despite preoperative counselling. They often continue smoking a week before or till the morning of surgery. Though smoking cessation and its impact on perioperative outcome are widely investigated worldwide we were unable to find any data regarding smoking cessation shortly before elective surgery in Pakistan.

The aim of this study was to determine the impact of the duration of smoking 
cessation before elective surgery on intraoperative hemodynamics, postoperative pain, and length of hospital stay. Post-operative pulmonary complications were also looked at.

\section{Methods}

It was a prospective cohort study conducted at the Aga Khan University Hospital Karachi, Pakistan after the approval of institutional ethical review committee (ERC reference No. 3948). A total of 260 patients were enrolled in the study after obtaining written informed consent. All adult patients aged 18 to 80 years of either sex with the history of smoking of a minimum of 6 months scheduled for elective non-cardiac intermediate to major surgery under general anaesthesia (with endotracheal intubation and controlled ventilation) were included.

Intermediate surgery includes open inguinal \& incisional hernia repairs, limb correction, and joint replacement surgeries while major surgery includes abdominal procedures with a standard midline incision, open major vascular surgery, and thoracic surgery with a standard unilateral thoracic incision.

Patients with American Society of Anaesthesiologist (ASA) physical status IV, $\mathrm{V}$ and patients scheduled for surgery under regional anaesthesia (spinal, epidural, transversus abdominis plane or TAP block and rectus sheet block, etc.) were excluded. Patients scheduled for minor surgery under general anaesthesia were also excluded. The voluntary participation, privacy \& confidentiality of each patient in the study were ensured and each one had the complete right of withdrawal from the study at any point.

Patients were recruited from the preoperative anaesthesia clinic through non-probability consecutive sampling method. Prior to taking consent, they were briefed about the objectives and public health impact of the study. As per routine, current guidelines of preoperative smoking cessation of about $4-8$ weeks before the scheduled surgery were discussed with each patient. Written informed consent was obtained from each participant by the trained data collectors. After obtaining consent, the patients were enrolled in the study and the scheduled date of surgery was recorded for step-wise data collection.

Patients were asked in the preoperative holding area regarding the duration of preoperative smoking cessation (exposure) in hours and/or days and were recorded. In operating room, standard monitors were applied before the start of Anaesthesia. These include electrocardiographs (ECG), non-invasive blood pressure (NIBP), oxygen saturation $\left(\mathrm{SPO}^{2}\right)$, end-tidal carbon dioxide $\left(\mathrm{ETCO}^{2}\right)$ and temperature monitoring. Other additional monitorings were also used depending on the type of surgery \& patient status. After routine preoxygenation, patients were induced with Propofol $(2-2.5 \mathrm{mg} / \mathrm{kg})$, atracurium $(0.5 \mathrm{mg} / \mathrm{kg})$, and tramadol $(1 \mathrm{mg} / \mathrm{kg})$ and trachea was intubated with an appropriate size endotracheal tube and put on controlled mode of ventilation. Intraoperative haemodynamic monitoring (heart rate, systolic blood pressure, diastolic blood pressure) was done at 16 different time points, i.e. before the induction of anaesthesia to ten minutes after the extubation. 
Postoperative analgesia was administered through either intermittent intravenous tramadol $50 \mathrm{mg} \mathrm{Q8H}$, tramadol intravenous infusion with a rate of 10 $15 \mathrm{mg} / \mathrm{kg}$ or tramadol patient controlled intravenous analgesia (PCIA) with initial setting of $10 \mathrm{mg}$ background infusion, 10 minutes of lockout time \& $10 \mathrm{mg}$ bolus. Adjuvant analgesics i.e. paracetamol $1 \mathrm{gm} \mathrm{Q8H}$ and injection ketorolac 30 $\mathrm{mg}$ Q8H were given if there was no contraindication. Postoperative pain score was monitored by visual analogue pain scale (VAS) of 0 - 10 scale (self-reporting by the patients) and record at four different timings. These included time at the arrival of PACU, three hours, six hours and 12 hours postoperatively.

Patients were also monitored for any major postoperative pulmonary complications i.e. pneumonia, pulmonary aspiration pneumonitis, acute lung injury (ALI), acute respiratory distress syndrome (ARDS). Need of non-invasive ventilation and/or invasive mechanical ventilation and length of stay in hospital were also recorded. Two trained data collectors were responsible for all data collection as per protocol and were supervised by principal investigator (PI) who did the random checks for the completeness and consistency of data collection forms. Dual entry was performed in two different computers to reduce the feeding error and then export to the statistical software for analysis.

\section{Statistical Analysis}

Analyses were conducted using Stata 12.0 (Stata Corp, College Station, TX). Quantitative and qualitative point estimates were summarized as mean \pm SD and frequency \& percentage respectively. Intraoperative hemodynamics parameter (heart rate, systolic BP, diastolic BP) and postoperative pain were response variables and four categories of preoperative smoking cessation duration were the main predictor.

We used Generalized Estimating Equation (GEE) to model intraoperative HR, systolic and diastolic BP choosing an unstructured correlation matrix structure. Binary logistic regression was used to observe the effect of preoperative smoking cessation duration on postoperative pain categories. To explore the confounding of BMI and duration of surgery were added to each model. The pain was also categorized into binary outcome (Mild and moderate) because severe pain was not observed and relative risk was reported with a $95 \%$ confidence interval. A P-value of 0.05 was considered statistically significant.

The sample size was calculated using STATA version 12.0 software. Fisher's z test of one correlation was used. 218 patients were required to achieve $85 \%$ power using a null correlation of -0.47 between smoking cessation and heart rate variability [9]. Assuming an alternate correlation between preoperative smoking abstinence and hear rate variation of -0.6 (one sided test), with a significance level of 0.05 . Adjusting for a $20 \%$ loss to follow up around 260 patients were recruited.

\section{Results}

A total of 260 patients fulfilling the eligibility criteria were recruited during the 
study period. Four patients were excluded due to consent refusal andata of 256 patients was included in the final analysis. Characteristics of patients, type of surgery, duration of smoking cessation and hospital stay are shown in Table 1. Group 1 has 143 patients (55.86\%), group 2, 53 patients (20.70\%), group 3, 29 patients (11.33\%) and group 4, 31 patients (12.11\%) according to duration of smoking cessation from less than 48 hours to more than 4 weeks (672 hours) (Table 1).

Effects of intraoperative heart rate, systolic and diastolic blood pressures were measured at sixteen different timings as hemodynamic variables. There was an average of $4.92 \approx 5$ beats/minute decrease in heart rate in patients who stopped smoking only for $\leq 48$ hours and this change is statistically significant as compared to patients who stopped smoking from 1 week to more than 4 weeks, i.e. reference category (Table 2).

There was an average of $6.81 \approx 7 \mathrm{mmHg}$ increases in systolic blood pressure in patients with preoperative duration of smoking cessation of $1-4$ weeks. This is a statistically significant change as compared to the patients with a duration of cessation of more than four weeks i.e. reference category (Table 2).

There was an overall decrease in diastolic blood pressure with different duration of smoking cessation. An average decrease of $6.05 \mathrm{mmHg}$ in patients with

Table 1. Baseline characteristics $(n=256)$.

\begin{tabular}{lc}
\hline \multicolumn{1}{c}{ Variables } & Point Estimates \\
\hline Age Groups & \\
18 - 65 years & $230(89.84)$ \\
65 - 80 years & $26(10.16)$ \\
Years of Smoking (Years) & $13.31 \pm 10.57$ \\
Smoking Intensity (Pack-year) & $10.25 \pm 14.69$ \\
Duration of Surgery (Minutes) & $141.95 \pm 91.98$ \\
Hospital stay (Days) & $2.85 \pm 1.32$ \\
Preoperative Smoking Abstinence & \\
Group 1: $\leq 48$ hours (2 days) & $143(55.86)$ \\
Group 2: $>48$ hours (2 days) - $\leq 168$ hours (7 days) & $53(20.70)$ \\
Group 3: $>$ 168 hours (1 week) - $\leq 672$ hours (4 weeks) & $29(11.33)$ \\
Group 4: $>672$ hours (4 weeks) & $31(12.11)$ \\
American Society of Anaesthesiologist (ASA) physical status & \\
ASA I & $49(19.14 \%)$ \\
ASA II & $163(63.67 \%)$ \\
ASA III & $44(17.19 \%)$ \\
Level of Education & \\
Less than Matriculation & $40(15.62)$ \\
Matric-Intermediate & $64(25.00)$ \\
Non-Professional education & $1(0.39)$ \\
Professional education & $151(58.98)$ \\
Type of surgery & \\
Intermediate & $163(63.67)$ \\
Major or complex & $93(36.33)$ \\
&
\end{tabular}


Table 2. Effect of preoperative smoking abstinence duration on intraoperative hemodynamic in simple regression models by generalized estimating equations.

\begin{tabular}{lcccccc}
\hline \multirow{2}{*}{$\begin{array}{c}\text { Preoperative Smoking } \\
\text { Abstinence in hours (days/weeks) }\end{array}$} & \multicolumn{2}{c}{ Heart Rate } & \multicolumn{2}{c}{ Systolic blood pressure } & \multicolumn{2}{c}{ Diastolic blood pressure } \\
\cline { 2 - 7 } & $\beta$ coefficient (SE) & $(95 \%$ CI) & $\beta$ coefficient (SE) & (95\% CI) & \multicolumn{1}{c}{$\beta$ coefficient (SE) } & (95\% CI) \\
\hline$\leq 48$ hours (2 days) & $-4.92(1.80)^{* *}$ & -8.45 to -1.39 & $2.76(2.17)$ & -1.50 to 7.02 & $-6.05(1.39)^{* *}$ & -8.78 to -3.31 \\
$>48.1-\leq 168$ hours (1 week) & $-3.47(2.05)$ & -7.50 to 0.55 & $0.28(2.48)$ & -4.58 to 5.15 & $-5.06(1.59)^{* *}$ & -8.18 to -1.93 \\
$>168-\leq 672$ hours (4 weeks) & $-0.02(2.34)$ & -4.62 to 4.57 & $6.81(2.83)^{*}$ & 1.25 to 12.37 & $-3.90(1.82)^{*}$ & -7.47 to -0.34 \\
$>672$ hours $(>4$ weeks) & \multicolumn{2}{c}{ Reference } & \multicolumn{2}{c}{ Reference } & Reference \\
\hline
\end{tabular}

${ }^{* *} \mathrm{p}<0.01 ;{ }^{*} \mathrm{p}<0.05 ; \mathrm{CI}=$ Confidence Interval; $\mathrm{SE}=$ Standard Error.

48 hours, $5.06 \mathrm{mmHg}$ decrease in 1 week and $3.90 \mathrm{mmHg}$ in 1 - 4 weeks duration of smoking cessation. All findings are statistically significant as compared to the patients with cessation of smoking more than 4 weeks i.e. reference category (Table 2).

Postoperative pain was measured at four different timings (PACU, 4 hours, 8 hours and 12 hours postoperatively) and categorized into mild pain (VAS pain score of $\leq 3$ ), moderate pain (VAS pain score of $>3$ to $\leq 6$ ) and severe pain (VAS pain score of $>6-10)$. None of the study patients developed severe pain in the first 12 hours postoperatively. The relative risk (RR) of mild to moderate pain in patients who stopped smoking for 48 hours at PACU and 3 hours postoperatively was 3.60 and 3.90 respectively and these are statistically significant as compared to the patients with duration of cessation of more than four weeks i.e. reference category as shown in table (Table 3).

Regarding postoperative complication, a total of 19 (7.42\%) patients developed minor respiratory complications including mild to moderate productive cough, sore throat and hoarseness of voice in the early postoperative period. None of the study patients developed major pulmonary complications including pneumonia, pulmonary aspiration pneumonitis, acute lung injury (ALI), acute respiratory distress syndrome (ARDS) and need for non-invasive ventilation and/or invasive mechanical ventilation. Three patients (1.17\%) had postoperative bleeding which was controlled after routine conservative management. Length of stay did not differ with duration of smoking cessation from 48 hours to more than 4 weeks.

\section{Discussion}

Smoking is considered an important risk factor for intra-operative and postoperative complications. Preoperative smoking cessation for four to eight weeks has shown to reduce overall peri-operative complications. Several studies have confirmed the impact of smoking on intraoperative hemodynamics and Postoperative Pain however the optimal duration of preoperative smoking cessation is not well established [10]. Our study reports that duration of cessation of smoking in majority of patients before surgery is 48 hours despite preoperative counselling of stopping smoking $4-8$ weeks This is significantly different from the 
Table 3. Effect of preoperative smoking abstinence duration on postoperative pain (mildmoderate).

\begin{tabular}{|c|c|c|c|}
\hline \multicolumn{4}{|c|}{ PAIN IN POST ANAESTHESIA CARE UNIT (PACU) } \\
\hline $\begin{array}{l}\text { PREOPERATIVE SMOKING } \\
\text { CESSATION (HOURS) }\end{array}$ & (Relative Risk) (Standard error) & $\begin{array}{l}\text { (95\% Confidence } \\
\text { Intervals) }\end{array}$ & (P-value) \\
\hline$\leq 48$ hours ( 2 days) & 1.58 & 1.52 to 8.51 & 0.003 \\
\hline$>48 \leq 168$ hours ( 2 days -1 week) & 1.18 & 0.88 to 6.28 & 0.088 \\
\hline$>168 \leq 672$ hours ( $1-4$ weeks) & 0.72 & 0.45 to 3.89 & 0.599 \\
\hline$>672$ hours (>4 weeks) & \multicolumn{3}{|c|}{ Reference category } \\
\hline & \multicolumn{3}{|c|}{ PAIN THREE HOURS POSTOPERATIVELY } \\
\hline $\begin{array}{l}\text { PREOPERATIVE SMOKING } \\
\text { CESSATION (HOURS) }\end{array}$ & (Relative Risk) (Standard error) & $\begin{array}{l}\text { (95\% Confidence } \\
\text { Intervals) }\end{array}$ & (P-value) \\
\hline$\leq 48$ hours ( 2 days) & 1.99 & 1.43 to 10.65 & 0.008 \\
\hline$>48 \leq 168$ hours ( 2 days -1 week) & 1.35 & 0.75 to 7.27 & 0.143 \\
\hline$>168 \leq 672$ hours $(1-4$ weeks $)$ & 1.33 & 0.39 to 4.48 & 0.642 \\
\hline$>672$ hours (>4 weeks) & \multicolumn{3}{|c|}{ Reference category } \\
\hline & \multicolumn{3}{|c|}{ PAIN SIX HOURS POSTOPERATIVELY } \\
\hline $\begin{array}{l}\text { PREOPERATIVE SMOKING } \\
\text { CESSATION (HOURS) }\end{array}$ & (Relative Risk) (Standard error) & $\begin{array}{l}\text { (95\% Confidence } \\
\text { Intervals) }\end{array}$ & (P-value) \\
\hline$\leq 48$ hours ( 2 days) & 1.65 & 0.77 to 9.02 & 0.122 \\
\hline$>48 \leq 168$ hours ( 2 days -1 week $)$ & 1.28 & 0.40 to 7.38 & 0.455 \\
\hline$>168 \leq 672$ hours ( $1-4$ weeks $)$ & 1.25 & 0.28 to 5.42 & 0.76 \\
\hline \multirow[t]{2}{*}{$>672$ hours (>4 weeks) } & \multicolumn{3}{|c|}{ Reference category } \\
\hline & \multicolumn{3}{|c|}{ PAIN 12 HOURS POSTOPERATIVELY } \\
\hline $\begin{array}{l}\text { PREOPERATIVE SMOKING } \\
\text { CESSATION (HOURS) }\end{array}$ & (Relative Risk) (Standard error) & $\begin{array}{l}\text { (95\% Confidence } \\
\text { Intervals) }\end{array}$ & (P-value) \\
\hline$\leq 48$ hours ( 2 days) & 0.67 & 0.11 to 4.00 & 0.669 \\
\hline$>48 \leq 168$ hours ( 2 days -1 week $)$ & 2.00 & 0.23 to 16.92 & 0.525 \\
\hline$>168 \leq 672$ hours ( $1-4$ weeks) & 0.75 & 0.098 to 5.69 & 0.781 \\
\hline$>672$ hours (>4 weeks) & \multicolumn{3}{|c|}{ Reference category } \\
\hline
\end{tabular}

Exposure; Preoperative Smoking cessation: $[\leq 48$ hours ( 2 days), $>48$ hours ( 2 days) $\leq 168$ hours ( 7 days), $>168$ hours (1 week) $\leq 672$ hours ( 4 weeks), $>672$ hours ( 4 weeks)]. Outcome; Postoperative pain: $[$ Mild pain $=1$, moderate pain $=0$ ] (Labels: Mild pain $=$ VAS Pain score $>0 \leq 3$, moderate pain $=$ VAS Pain score $>3 \leq 6$ )

previously reported duration of cessation ranging from 2 weeks to 8 weeks in elective surgery [6]. It was thought that the most likely reasons were cultural factors, literacy level and inadequate counselling in preoperative clinics related to the recommended smoking cessation period for elective surgeries. Our data negated the impact of literacy level on the compliance of preoperative advice; especially related to smoking cessation. We found that about $55.86 \%$ of patients did not stop smoking as per advice irrespective of their literacy level [6]. 
The haemodynamic key findings of this study include 1) decreased intraoperative heart rate in patients with a preoperative smoking cessation of less than or equal to 48 hours 2) significant rise in systolic blood pressure in the category of patients with a preoperative smoking cessation of one week to four-week duration 3) fall in diastolic blood pressure with increasing preoperative smoking cessation duration.

The exact reason for decreasing heart rate remained unclear however the cardio-depressant effect of anaesthetic agents may be enhanced among smokers with a preoperative cessation of fewer than 48 hours. Another possible reason is related to the lack of sympathomimetic effect of nicotine with general depressant effect [11]. Nicotine has a half-life of around 30 minutes and its active metabolites may remain in the body for around 20 hours [11]. Several studies in past have focused on the heart rate changes with smoking duration with conflicting results [12] [13].

The diastolic blood pressure has an inverse relationship with increased duration of preoperative smoking cessation i.e. highest drop in blood pressure in patients with the shortest duration of smoking cessation. In contrast, systolic blood pressure significantly increased in patients for one week to four-week duration and the effect was statistically insignificant with smoking cessation of fewer than 48 hours and more than four weeks. Our study findings conclude that even a short duration of smoking cessation may confer some benefit considering the acute effects of nicotine and carbon monoxide on the cardiovascular system. The earlier study also showed that the increased heart rate and blood pressure were enhanced in male gender as compared to female [14]. As we could only recruit seven female patients in our study, so the precise effect of gender on patients' haemodynamics could not be analyzed.

The results of this study also showed that none of the study patients developed severe pain in early i.e. 12 hours postoperatively and mean postoperative pain score was less in PACU and first post-operative reading in ward at 4 hours in patients with preoperative smoking cessation of fewer than 48 hours. The analgesic effect of nicotine in this group of patients is perhaps responsible for better pain control as per VAS. It is likely that with increasing duration of smoking cessation (i.e. after six hours to more than 4 weeks), nicotine clears off from plasma gradually and has shown reduced nociceptive effect. In one study by Chiang HL et al. 2016, they compared three groups of patients for postoperative pain scores measured by VAS, 1) Non-smokers, 2) Current smokers and 3) Past smokers [15]. They concluded that on the first postoperative day, the dynamic pain score was insignificant between the non-smoker and current smoker groups $5.1 \pm 1.8$ Vs $5.6 \pm 1.8$ (Adjusted $\mathrm{P}=0.090$ ) respectively [15]. It was also found insignificant when compared the current smokers with past smokers $5.6 \pm 1.8 \mathrm{Vs}$ $5.0 \pm 1.8$ (Adjusted $\mathrm{P}=0.096$ ) respectively [15]. In our study, we only included current smokers and pain was measured by VAS on more frequent time points. Therefore, within current smokers, the pain perception findings were more precisely elaborated. 
In our study the exposure to the smoking was self-reported. This is the limitation of the study as self-reported smoking cessation may not be a reliable method of exposure measurement and the estimated effect of smoking cessation on the intraoperative haemodynamics and early postoperative pain may be over or underestimated. In a subsequent study, we are planning to validate the self-reported smoking abstinence with the salivary nicotine levels.

Another limitation of the study was the recruitment of very few female smokers. The reported prevalence of smoking among female "general population" was around 9\% in Karachi, Pakistan. However, the actual prevalence specifically among "female surgical patients" is still unknown. We could only capture female patients around $3 \%$ of the total sample while around $97 \%$ were males. Due to this imbalance in the cohort, the effect of gender as a confounder cannot be evaluated. To our knowledge, this is the first study that has emphasized the perioperative effects secondary to different durations of short-term preoperative smoking abstinence among current smokers. The findings of this study have implications for clinical practice in Pakistan and will also help in developing some local or regional guidelines related to preoperative smoking cessation. This study also emphasized the need for proper counseling of patients about the perioperative hazards of smoking and the importance of timely smoking abstinence before surgery.

\section{Conclusion}

Preoperative smoking cessation has a significant association with intraoperative haemodynamics and early postoperative pain scores. It is likely that even a relatively short period of smoking cessation helps to avoid some of the adverse effects and reduces perioperative complications so smoking cessation should be encouraged at any time. Preoperative assessment and counseling of patients for smoking cessation before elective surgery are the essential components of safe clinical practice.

\section{Acknowledgements}

- I acknowledge this to Almighty ALLAH.

- My family.

- Thesis Supervisor \& co-investigator; Dr. Gauhar Afshan.

- Committee Members; Dr. Muslima Ejaz, Mr. Syed Iqbal Azam.

- Mr. Akbar Shaukat \& Mr. Muhammad Ali; Data collection and data entry.

\section{Funding Sources}

1) Anaesthesia Department Research Grant (Departmental Research Grant).

2) Institutional Seed Money Grant.

\section{Conflicts of Interest}

The authors declare no conflicts of interest regarding the publication of this paper. 


\section{References}

[1] Ahmed, R., Rizwan-ur-Rashid, M.P. and Ahmed, S.W. (2008) Prevalence of Cigarette Smoking among Young Adults in Pakistan. Journal of Pakistan Medical Association, 58, 597-601.

[2] Maher, R. and Devji, S. (2002) Prevalence of Smoking among Karachi Population. Journal of Pakistan Medical Association, 529, 250-253.

[3] Khan, F.S., Lotia-Farrukh, I., Khan, A.J., Siddiqui, S.T., Sajun, S.Z., Malik, A.A., et al. (2013) The Burden of Non-Communicable Disease in Transition Communities in an Asian Megacity: Baseline Findings from a Cohort Study in Karachi, Pakistan. PLoS ONE, 8, e56008. https://doi.org/10.1371/journal.pone.0056008

[4] Wong, J., Lam, D.P., Abrishami, A., Chan, M.T. and Chung, F. (2012) Short-Term Preoperative Smoking Cessation and Postoperative Complications: A Systematic Review and Meta-Analysis. Canadian Journal of Anesthesia, 59, 268-279. https://doi.org/10.1007/s12630-011-9652-x

[5] Cuvas, O., Er, A., Ikeda, O., Dikmen, B. and Basar, H. (2008) Cigarette Smoking and the Haemodynamic Response to Tracheal Intubation. Anaesthesia, 63, 463-466. https://doi.org/10.1111/j.1365-2044.2008.05451.x

[6] Myers, K., Hajek, P., Hinds, C. and McRobbie, H. (2011) Stopping Smoking Shortly before Surgery and Postoperative Complications: A Systematic Review and Meta-Analysis. Archives of Internal Medicine, 171, 983-989. https://doi.org/10.1001/archinternmed.2011.97

[7] Theadom, A. and Cropley, M. (2006) Effects of Preoperative Smoking Cessation on the Incidence and Risk of Intraoperative and Postoperative Complications in Adult Smokers: A Systematic Review. Tobacco Control, 15, 352-358. https://doi.org/10.1136/tc.2005.015263

[8] Roizen, M.F. and Fleisher, L.A. (2009) Anesthetic Implications of Concurrent Diseases. In Miller Anesthesia, 7th Edition, Saunders, USA.

[9] Turan, A., Mascha, E.J., Roberman, D., et al. (2011) Smoking and Perioperative Outcomes. Anesthesiology, 114, 837-846. https://doi.org/10.1097/ALN.0b013e318210f560

[10] Shi, Y., Weingarten, T.N., Mantilla, C.B., Hooten, W.M. and Warner, D.O. (2010) Smoking and Pain Pathophysiology and Clinical Implications. Anesthesiology, 113, 977-992. https://doi.org/10.1097/ALN.0b013e3181ebdaf9

[11] Carrick, M.A., Robson, J.M. and Thomas, C. (2019) Smoking and Anaesthesia. BJA Education, 19, 1-6. https://doi.org/10.1016/j.bjae.2018.09.005

[12] Barutcu, I., Esen, A.M., Kaya, D., Turkmen, M., Karakaya, O., Melek, M., et al. (2005) Cigarette Smoking and Heart Rate Variability: Dynamic Influence of Parasympathetic and Sympathetic Maneuvers. Annals of Noninvasive Electrocardiology, 10, 324-329. https://doi.org/10.1111/j.1542-474X.2005.00636.x

[13] Møller, A.M., Villebro, N., Pedersen, T. and Tønnesen, H. (2002) Effect of Preoperative Smoking Intervention on Postoperative Complications: A Randomised Clinical Trial. The Lancet, 359, 114-117. https://doi.org/10.1016/S0140-6736(02)07369-5

[14] Thomsen, T., Villebro, N. and Møller, A.M. (2014) Interventions for Preoperative Smoking Cessation. Cochrane Database of Systematic Reviews, 2014, Article ID: CD002294.

[15] Chiang, H.-L., Chia, Y.-Y., Lin, H.-S. and Chen, C.-H. (2016) The Implications of Tobacco Smoking on Acute Postoperative Pain: A Prospective Observational Study. Pain Research and Management, 2016, Article ID: 9432493. https://doi.org/10.1155/2016/9432493 